\title{
Health care services use among long-term breast cancer survivors: a systematic review
}

\author{
Anna Jansana 1,2,3 - Margarita Posso ${ }^{1,3}$ - Inmaculada Guerrero ${ }^{4}$ - Alexandra Prados-Torres ${ }^{3,4}$. \\ Maria Isabel Del Cura ${ }^{3,5,6} \cdot$ Xavier Castells ${ }^{1,3,7}$. Maria Sala ${ }^{1,3,7}$ (D)
}

Received: 21 August 2018 / Accepted: 21 March 2019

(C) The Author(s) 2019

\begin{abstract}
Purpose Long-term breast cancer survivors are women surviving at least 5 years after diagnosis. This systematic review aimed to summarize the main characteristics and patterns of healthcare service use (frequency of visits, health providers visited, and preventive care performed) among long-term breast cancer survivors.

Methods We used standard Cochrane Collaboration methods and searched the MEDLINE and EMBASE databases up to January 2018. We included English language observational studies reporting health service use among long-term cancer survivors. The quality of the studies was appraised through the ROBINS-I assessment tool. Two independent reviewers performed both the study quality assessment and the data extraction.

Results A total of 23 observational studies were included that reported data on health services use by long-term breast cancer survivors. Despite heterogeneity among studies, about half of them reported that breast cancer survivors visited a medical provider at least once a year, as recommended by breast cancer survival guidelines. Although survivors visited medical providers with the recommended frequency, a substantial number used specialist care instead of primary care during follow-up. The results showed underuse of the recommended annual mammogram.

Conclusions Long-term breast cancer survivors differ in their health services use with regard to the frequency of visits as well as the health providers seen. Our results indicate the need for active surveillance through primary care providers in coordination with specialized care.

Implications for cancer survivors This review could help to standardize the management of breast cancer survivors and decisionmakers to adapt their guidelines and clinical protocols.
\end{abstract}

Keywords Breast cancer · Survivorship $\cdot$ Health services $\cdot$ Preventive care $\cdot$ Mammography

Electronic supplementary material The online version of this article (https://doi.org/10.1007/s11764-019-00755-z) contains supplementary material, which is available to authorized users.

Maria Sala

msalaserra@parcdesalutmar.cat

1 Department of Epidemiology and Evaluation, IMIM (Hospital del Mar Medical Research Institute), Passeig Marítim, 25-29, 08003 Barcelona, Spain

2 European Higher Education Area (EHEA) Doctoral Programme in Methodology of Biomedical Research and Public Health in Department of Pediatrics, Obstetrics and Gynecology, Preventive Medicine and Public Health, Universitat Autónoma de Barcelona (UAB), Bellaterra, Barcelona, Spain

3 Research Network on Health Services in Chronic Diseases (REDISSEC), Barcelona, Spain
4 Grupo EpiChron de Investigación en Enfermedades Crónicas, IACS, IIS Aragon, Unidad Docente de Medicina Preventiva y Salud Pública, Hospital Universitario Miguel Servet, Paseo Isabel La Católica 1-3, 50009 Zaragoza, Spain

5 Primary Care Research Unit, Gerencia de Atención Primaria, Madrid, Spain

6 Department of Preventive Medicine and Public Health, University Rey Juan Carlos, Avenida de Atenas, 28922 Alcorcón, Madrid, Spain

7 Autonomous University of Barcelona (UAB), 08193 Bellaterra, Barcelona, Spain 


\section{Introduction}

Long-term breast cancer survivors are those women who survive free from cancer recurrence or new primary cancer for at least 5 years [1]. It is well-known that survival has increased in women with breast cancer, particularly in European countries as well as in the USA, and trends suggest that it will keep increasing. Recent data from the NORDCAN Project show that around $87 \%$ of women diagnosed with breast cancer in the Nordic countries will live disease-free for at least 5 years and $74-83 \%$ for at least 10 years [2], which is slightly higher than in the rest of Europe. In the USA, the 5-year relative survival rate for all types of breast cancer was $90 \%$ in 2014 [3].

In 2011, the survival rate for breast cancer ranked fifth for all cancers and third when analyzing only women [4]. The increased likelihood of survival can be explained by numerous factors, such as improvements in breast cancer screening and advances in diagnosis and treatment and aging $[5,6]$. Due to the increase in survivorship rates and life expectancy among breast cancer survivors, the pool of long-term breast cancer survivors is likely to expand considerably in the coming decades [7-10].

Cancer survivors' follow-up is an essential phase of cancer care and includes surveillance, symptom control interventions, comorbidities, and complications treatment, as well as psychosocial needs and care coordination mechanisms. This is usually performed by both the primary care providers (GP, nurses, social workers) and the specialized attention (medical oncologist, radiation oncologist, gynecologists, physiotherapist, psychologist) $[8,11,12]$.

The critical point in survival care appears after the discharge from oncology or specialist care, when these women remain at long-term risk of complications due to treatment and the risk of relapses [13]. However, it is not clear whether women who have received cancer therapy are more likely to develop other comorbidities than those without a history of cancer [14]. Because of this, it is essential that breast cancer survivors, besides breast cancer specific prevention, receive the standardized preventive care as the women without cancer history. Furthermore, clear guidelines are needed to assist decision-making on health care planning and follow-up in this group of women and to improve the efficiency of primary care officers [1].

The National Cancer Institute from USA sets among their six priorities, for cancer-related public health research, to study how to meet the needs and challenges of a growing population of cancer survivors [15]. It is essential to provide evidence on how health systems should answer to the longterm survivors' follow-up. The use of healthcare services by breast cancer survivors presents new challenges for health organizations in terms of the role of every health professional and the characteristics and frequency of visits and tests performed. While there are many evidence-based guidelines for diagnosis and treatment, there are far fewer for long-term survivorship follow-up [16-18] and, particularly, there is little evidence on the type of follow-up and health needs of this population group [17-23]. Evidence synthesis methods are an excellent alternative for decision-makers to assess the scope, quality, and relevance of available data and to identify areas of clinical uncertainty and gaps in available scientific information.

This systematic review aimed to describe the main characteristics and patterns of healthcare services use (frequency of visits, health providers visited, and preventive care performed) among long-term breast cancer survivors.

\section{Methods}

\section{Study design and search strategy}

This systematic review was conducted following standard Cochrane Collaboration methods [24] with a predetermined review protocol registered in the PROSPERO database (CRD42018089309). We searched MEDLINE and EMBASE databases from their inception to 24 January 2018, following the PRISMA statement [25], to obtain all the existing literature on health services use and preventive care in long-term breast cancer survivors. Health services use was assessed through the frequency of visits to primary care providers, specialists and other health professionals, and the main reason to visit. Preventive care included all tests performed in women designed to identify a new incident cancer or relapse and all the surveillance tests for cancer-related reasons or not.

The search strategy included a combination of controlled vocabulary and search terms related to long-term breast cancer survivors and health services use, such as breast cancer, cancer survivor, survivorship, health services use, health care utilization, general practitioner, specialist, and follow-up care. The search strategy can be found in Appendix A. Stage I screening identified titles and abstracts related to the main objective and was done by two independent reviewers. Stage 2 screened the full-text articles against the inclusion and exclusion criteria, described below. Articles meeting the inclusion criteria were included in the synthesis; this phase was also conducted by two reviewers. In all cases, agreement was reached after discussion of any differences. The results of this process are reported in a PRISMA flowchart (Fig. 1).

\section{Inclusion and exclusion criteria}

Inclusion was limited to observational studies written in English. Systematic reviews were used as the source of primary studies. The included studies were published between 2006 and January 2018. Studies reporting the frequency of health care use in long-term breast cancer survivors, defined as the period starting 5 years after diagnosis, were included. 
Fig. 1 PRISMA flowchart illustrating study inclusion

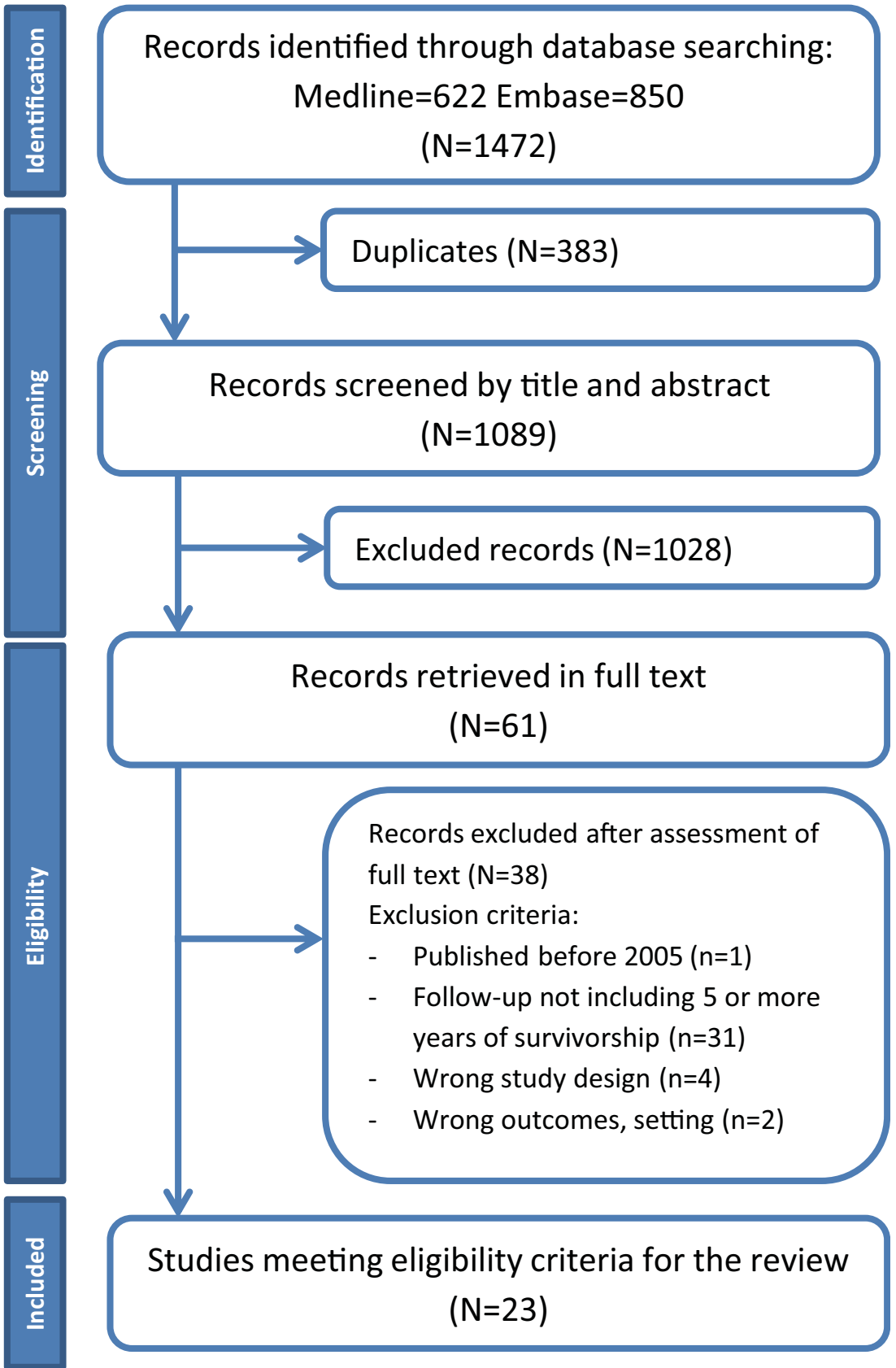

We excluded narrative reviews, conference abstracts, recommendations, summary reports, and editorials. Studies not providing detailed information by survival period (at least 5 years after diagnosis) were excluded, as were studies about cancer in general that did not provide results for breast cancer specifically. Studies published before 2005 were excluded, as the treatment for breast cancer changed then because of the introduction of trastuzumab therapy. Treatment with this trastuzumab in HER2-positive tumors at an early stage improved overall survival and disease-free survival [26]. Lastly, studies aiming to analyze patients' and health care providers' satisfactions with healthcare were also excluded unless they reported data on health services use.

\section{Quality assessment and data extraction}

We developed a predefined form to extract information from included studies. The following information was selected: author, country, year of publication, study design, purpose, population characteristics, survival period, type of analysis, results by outcomes (health services use and preventive care), data source, and study limitations. Two reviewers independently extracted the data for the included studies. Disagreements between them were resolved by consensus.

To assess the methodological quality of the studies, we used the Risk of Bias in Non-randomized Studies of Interventions (ROBINS-I) assessment tool [27]. The following sources of bias 
were assessed: (i) bias due to confounding, (ii) bias in the selection of participants into the study, (iii) bias due to missing data, (iv) bias in measurement of outcomes, (v) bias in the selection of the reported result, and (vi) overall bias. For each study, the risk of bias for each domain was rated as low risk of bias, moderate risk of bias, serious risk of bias, critical risk of bias, or no information. The final risk of bias assessment was based on consensus by two reviewers following the ROBINS guidelines [27] and was considered in the interpretation of the results.

\section{Analysis and synthesis of the results}

There was wide heterogeneity across study populations and diversity in the categories of survivor follow-up among the studies. This and the way the results were analyzed and presented in the studies precluded the possibility of pooling the data across studies. Therefore, we performed a narrative synthesis with tabulation of the characteristics and main outcomes of the included studies. The studies are ordered according to study design in the tables as follows: retrospective cohort with a control group, retrospective cohort without a control group, and cross-sectional studies.

\section{Results}

A total of 1089 abstracts were eligible for title and abstract screening, and 61 were checked at the full-text reading. Twenty-three observational studies met the eligibility criteria and were included in the final review. Of them, 12 (52.2\%) were retrospective cohort studies without a control group, 6 (26.1\%) were retrospective cohort studies with a control group, and 5 $(21.7 \%)$ were cross-sectional studies. The publication period ranged from 2005 to 2018, and 9 studies (39\%) were conducted in the USA, 11 (48\%) in Europe, of which 3 (13\%) were from the UK. The data source was tumor registries or hospital databases in $12(52.2 \%)$ of the included studies, self-reported surveys or interviews in $9(39.1 \%)$ studies, and $2(8.7 \%)$ used both sources.

\section{Population characteristics}

The 23 studies involved 476,127 women aged 18 years or older with different parameters (Table 2). The period of breast cancer diagnosis varied from $1989[34,47]$ to 2013 [44, 46]. In terms of location, eight studies (34.8\%) included populations from both urban and rural areas, and $3(13 \%)$ included only urban areas, while $12(52.2 \%)$ did not report characteristics regarding location. To assess comorbidities during follow-up, 4 (17.4\%) of the 23 articles used the Charlson comorbidity index, 6 (26.1\%) did not report information about comorbidities, and the rest included a list of different diseases associated with breast cancer treatment. More details on comorbidities are shown in Table 1.

The range of reported survival periods differed among all the studies, even though all of them included survival of at least 5 years or more. Lu et al. [39] included the longest follow-up period (16 years from diagnosis), followed by the studies by Maddams et al. [40] and Weaver et al. [49] with 16 and 14 years of follow-up, respectively.

\section{Outcomes}

To summarize the outcomes, we performed a tabulation of the given measures and risk estimators (Table 2). A total of 10 (44\%) studies described both healthcare use and preventive care, $9(39 \%)$ described healthcare utilization, and $4(17 \%)$ described preventive health practices. No differences were found in terms of reported outcomes by country.

\section{Health services use}

Eleven studies reported that breast cancer survivors visited a primary care provider or a specialist at least once a year [28, 32, 33, 35, 37-39, 41, 44, 46, 50]. Some studies reported more visits to specialist care, such as an oncologist, surgeon, radiotherapist, gynecologist, or other specialist [28, 32, 49], while another three studies reported more visits to primary care physicians than to specialist care $[35,37,38]$. No differences were found between the studies reporting more visits to specialized attention compared to those reporting more to primary care in terms of country, publication period, years of follow-up, period of diagnosis, or data source. Another study reported that $58.8 \%$ of the patients used complementary therapies after treatment completion [32].

Regarding the reason for consultation, two studies agreed that the main reason for breast cancer survivors visiting the health services was non-cancer-related issues and that the medical provider most frequently consulted for these health problems was the primary care provider [32, 42]. In four studies, the predictors of health services use were lower well-being score, having had a mastectomy, having two or more comorbidities, having a painful arm, and younger age [34, 39, 43, 48]. Even so, only two of these studies analyzed both primary care and specialized attention [43, 48] and attributed the highest use to comorbidities management in younger survivors. In contrast, one study showed that the oldest survivors ( $>70$ years) had twice the probability of visiting a physician in the last 3 months rather than their younger counterparts. Nevertheless, that study did not adjust by comorbidities and was focused only in the specialized attention without taking into account the use of health services related with age and preventive care [44].

In terms of the trends in long-term use, three studies showed that the average number of visits decreased over time [29, 35, 49], but other studies did not find similar results [42] or found that this decrease occurred only in specialist care but not in primary care physician visits [30]. However, only two studies included age-adjusted models [30, 49].

Five studies compared health services use between survivors and the general population. The likelihood of using the 


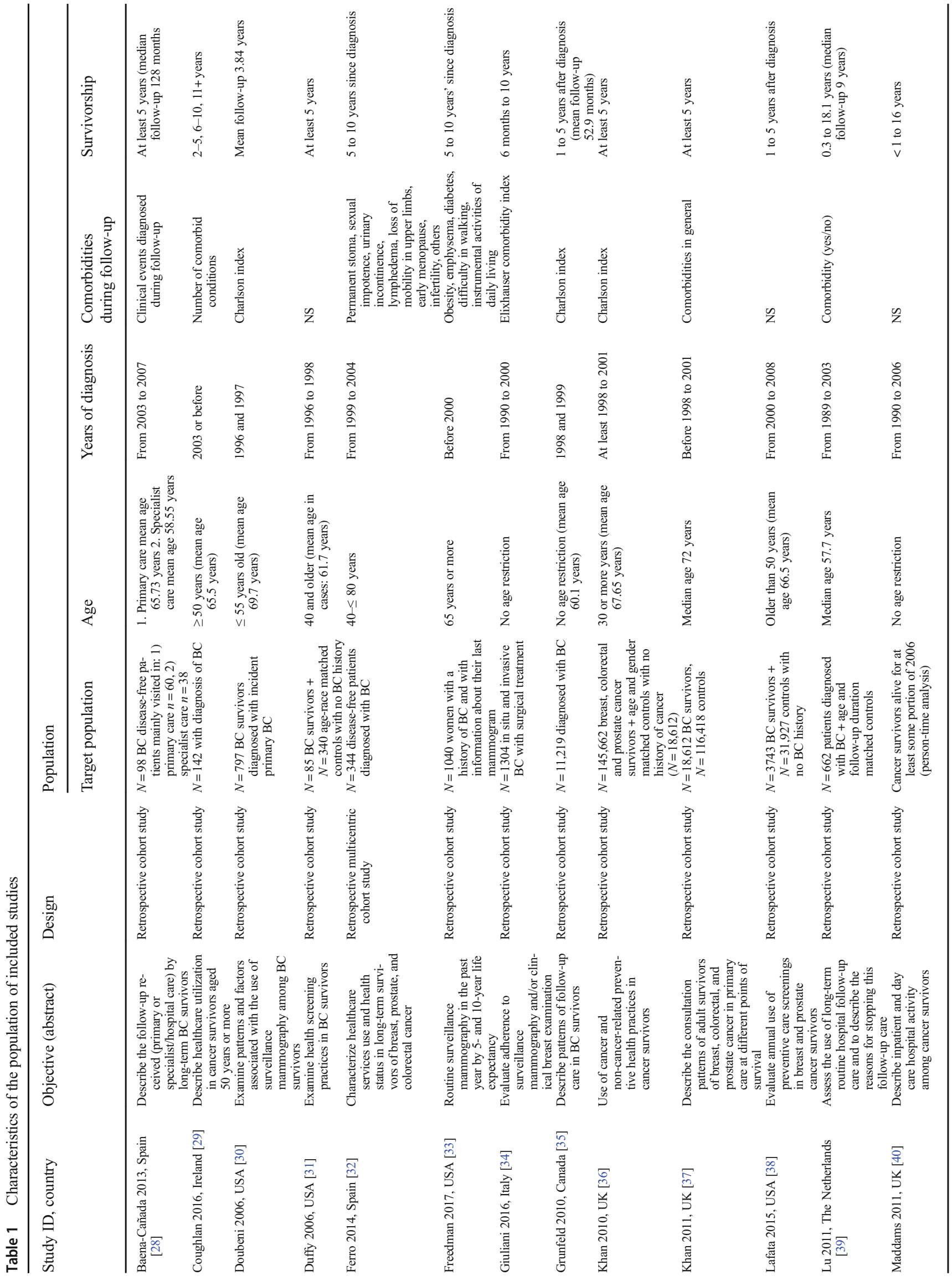




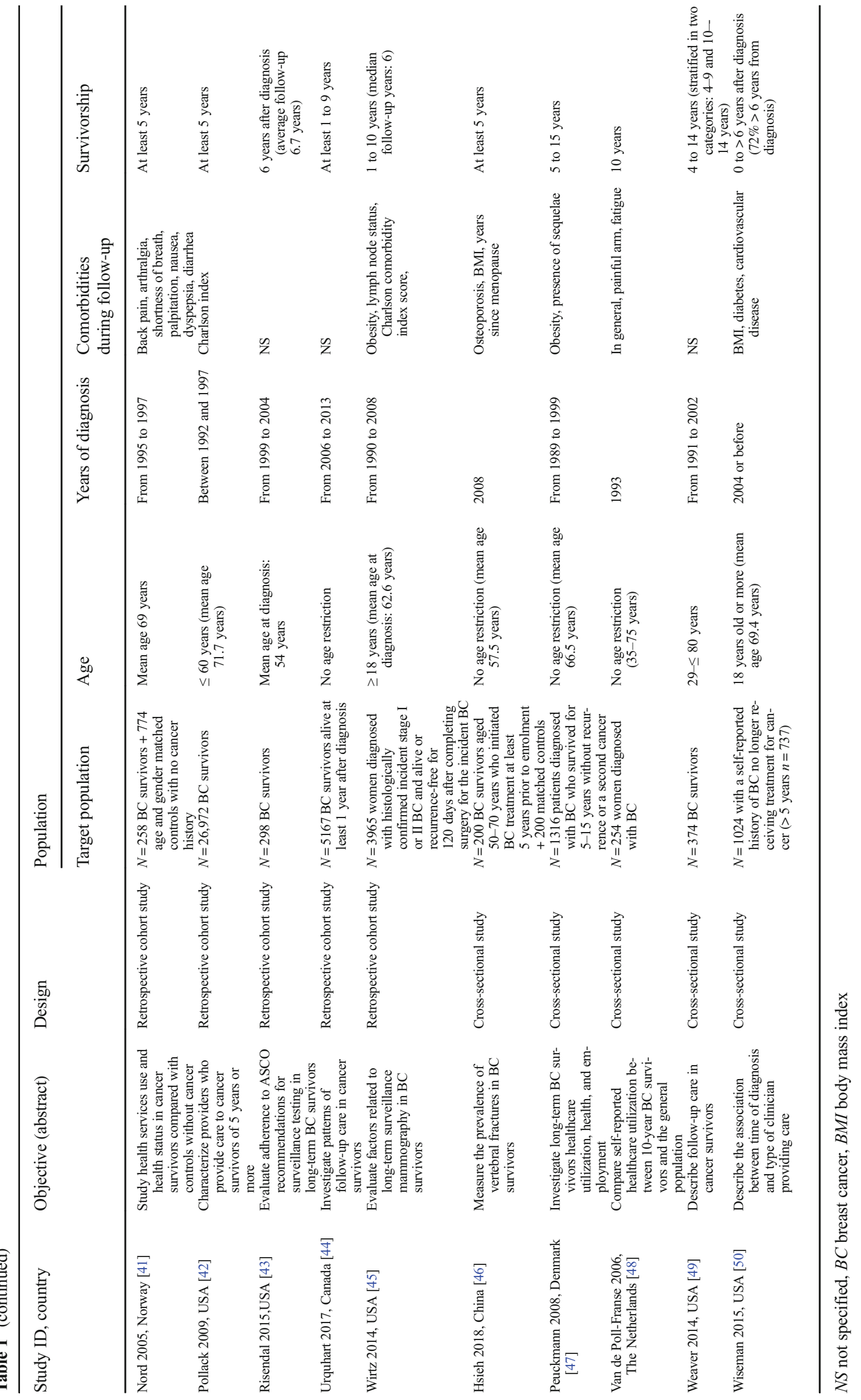




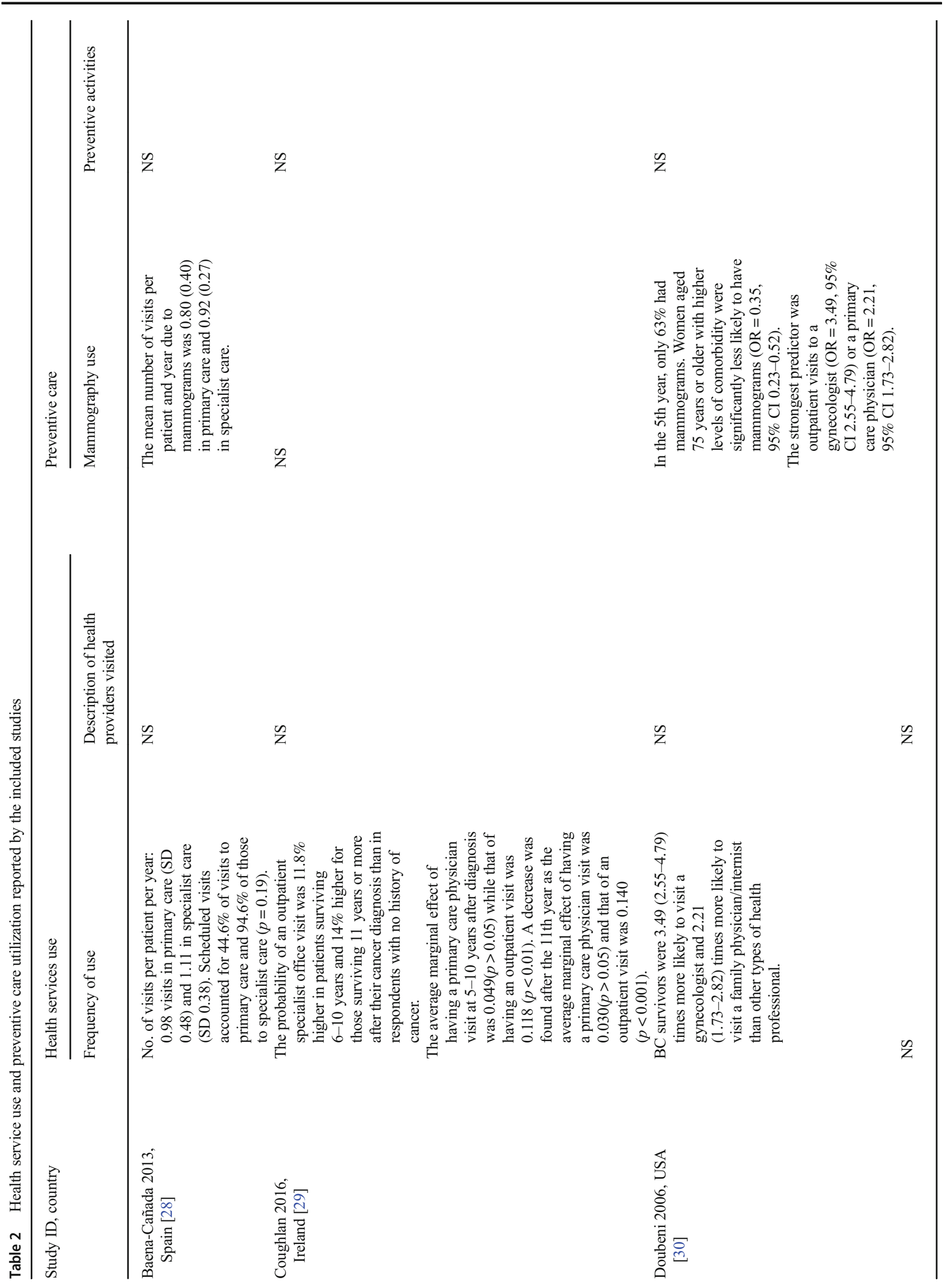




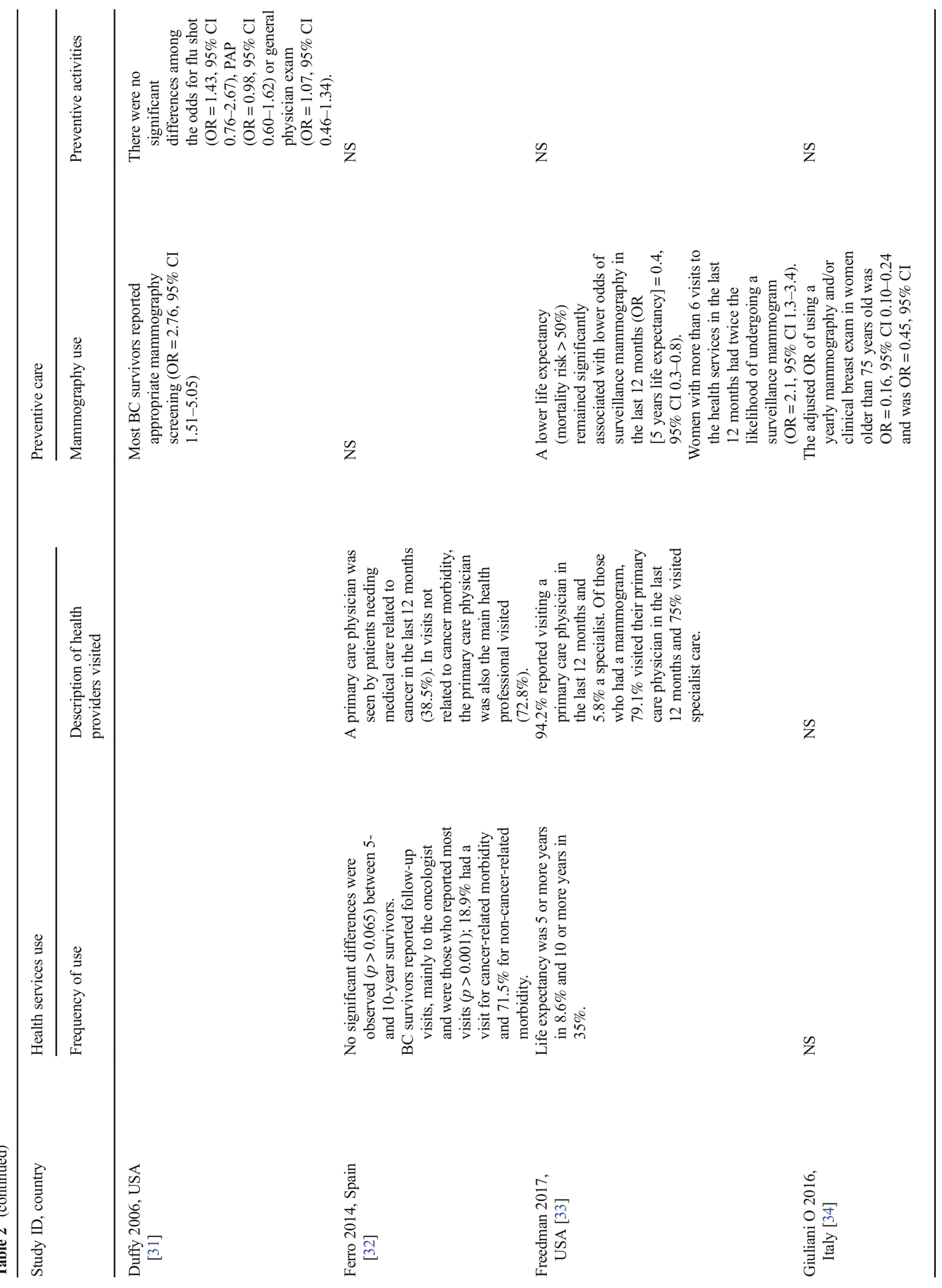




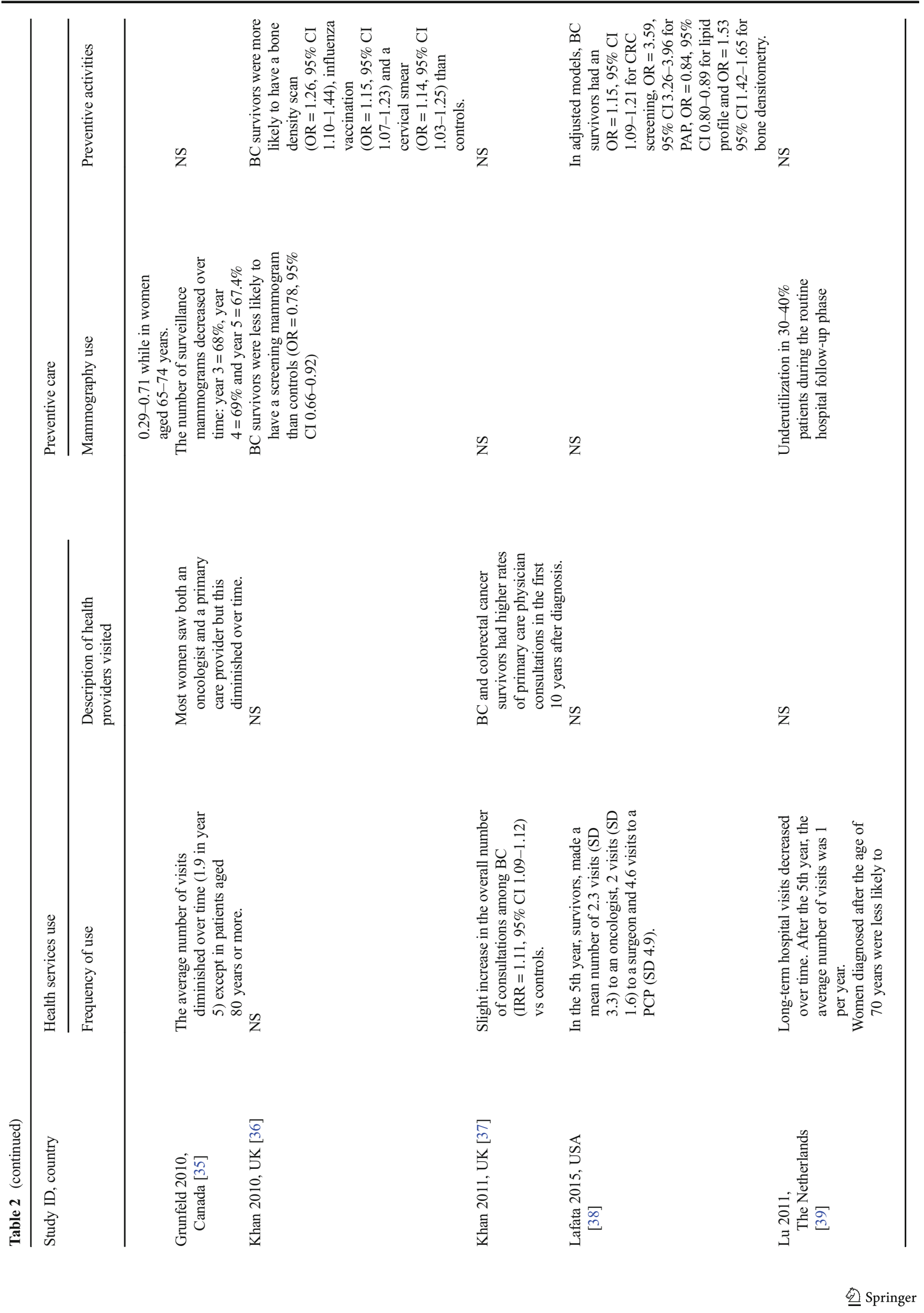




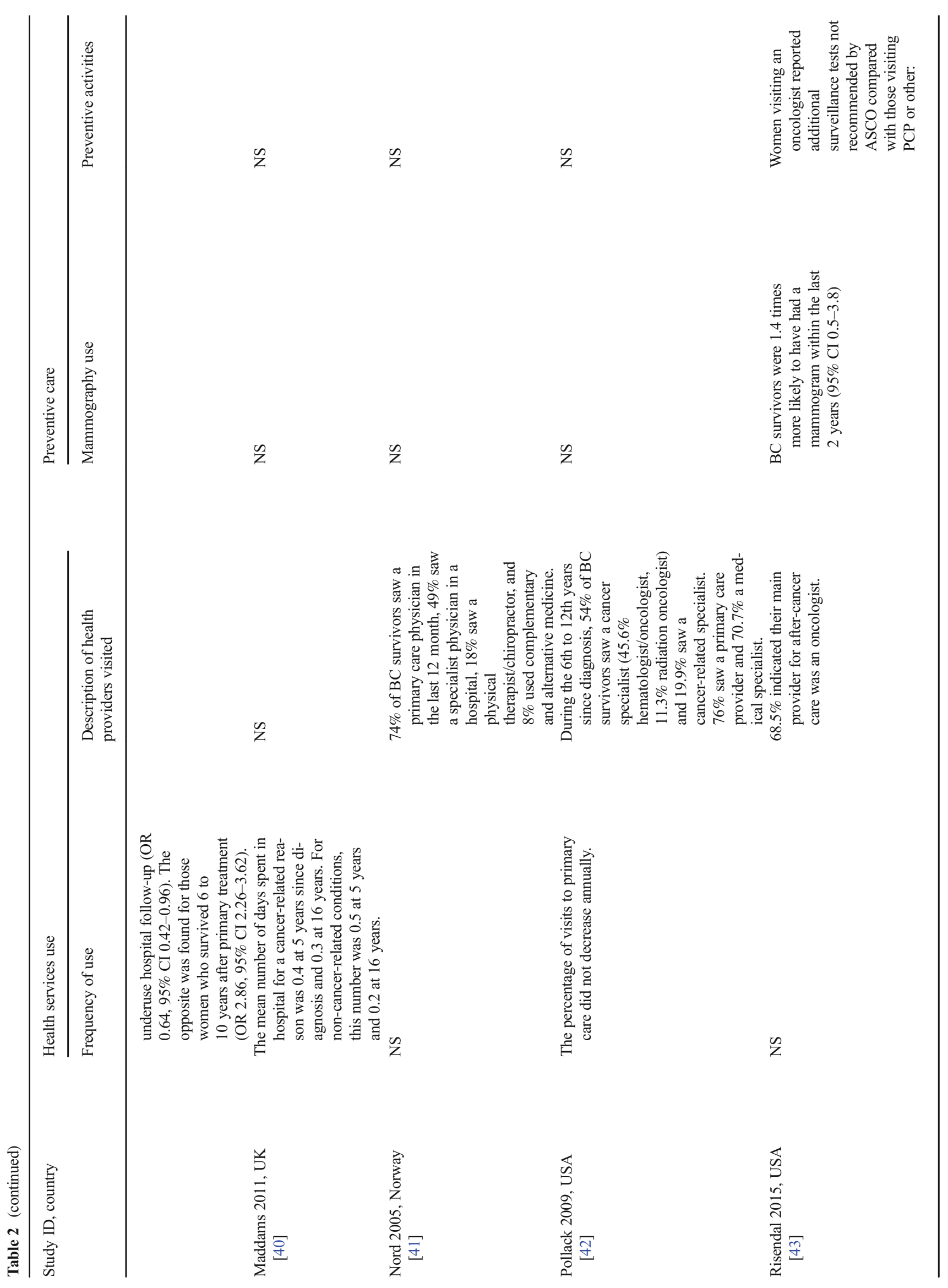




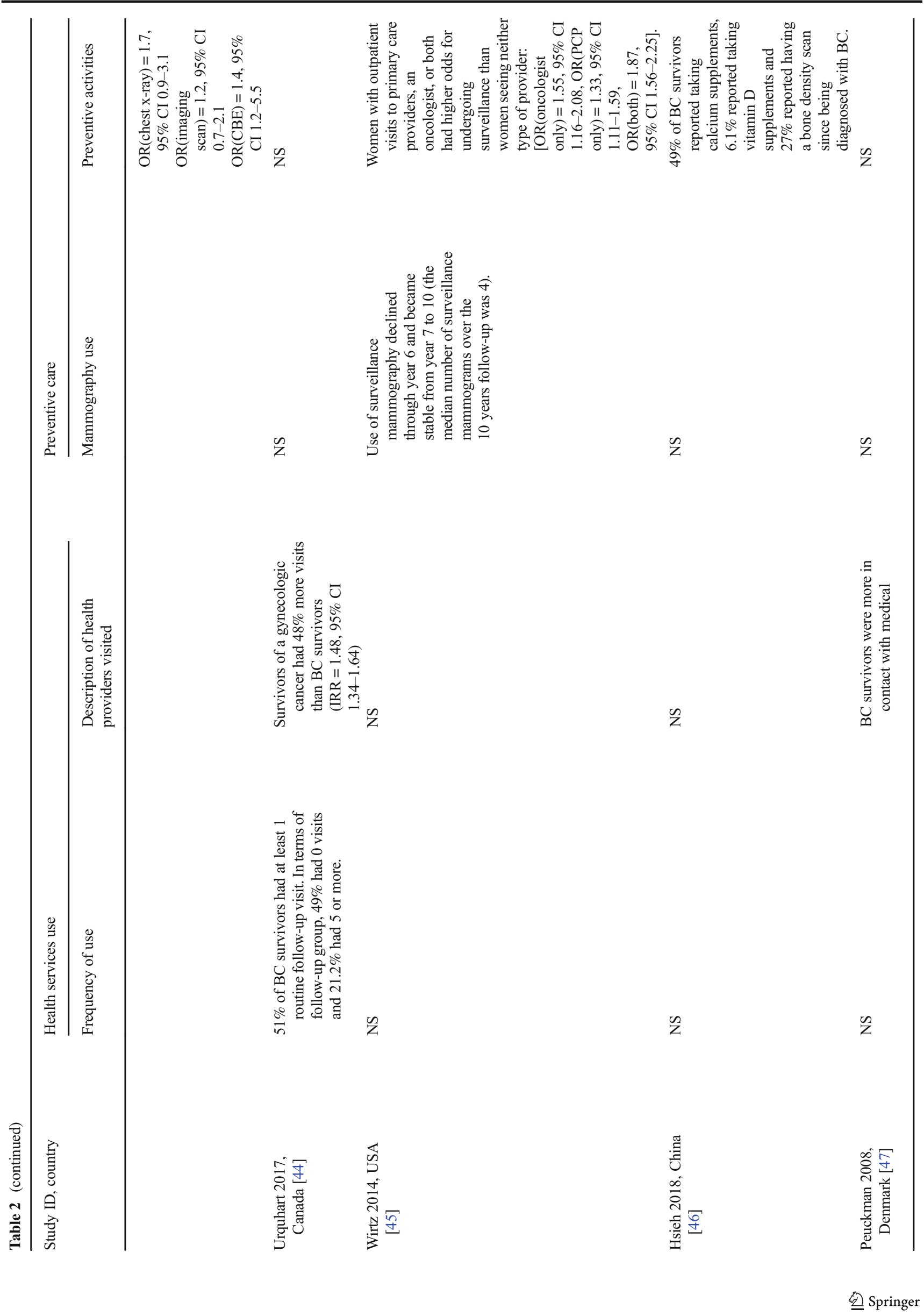




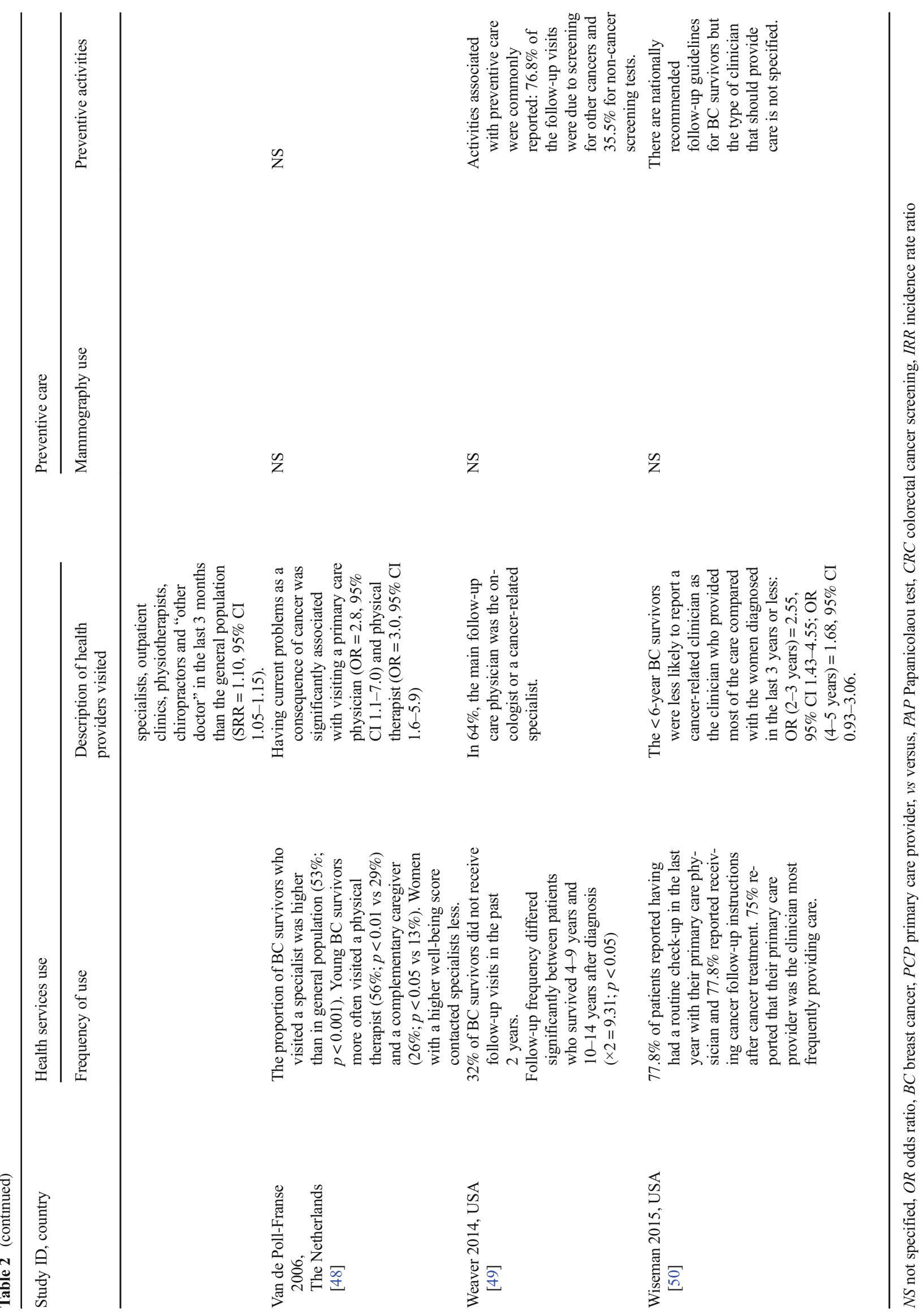


health services in breast cancer survivors compared to the general population ranged from 1.1 (95\% CI 1.09-1.12) [37] to 1.48 (95\% CI 1.34-1.64) [47]. Two studies agreed that this increase occurred only in specialist care $[31,36]$ and in the use of complementary therapies [41]. The percentages of breast cancer survivors using complementary therapies was $26 \%$ in 10 -year survivors and $13 \%$ in their matched controls [48].

\section{Preventive care}

Mammograms were the most widely studied preventive activity in this population. Mixed results were found in its utilization. Two study showed that mammography screening was performed according to the guidelines in breast cancer survivors (once a year from the fifth year of survivorship) [28, 31], while other four studies found underutilization regarding the annual mammography screening recommended for breast cancer survivors [35, 39, 43, 45]. Two studies reported that screening mammography was more likely to be performed in breast cancer survivors than in controls. It should be noticed that recommendations for the two populations differ [31, 43].

The main predictor for mammography use was visits to the health services [28, 43, 45]. Specifically, mammography is more frequently recommended by oncologists rather than by primary care providers or other health specialists [28, 43]. Being older with high levels of comorbidities was associated with a lower probability of undergoing mammography [30, $31,33,35,47]$.

Five studies [30, 38, 43, 46] evaluated imaging scans. More imaging tests were reported in breast cancer survivors rather than in the general population, specifically bone density scans $[31,36,38]$. In addition, those women whose main medical provider was an oncologist were more likely to undergo additional imaging scans [43].

In terms of general preventive activities, breast cancer survivors were more likely to receive appropriate preventive screening than controls for both cancer and non-cancer screening $[31,38,49]$. Another study found similar results but pointed out that when the main medical provider was the oncologist, breast cancer survivors received additional surveillance tests not recommended by the guidelines [28, 43, 49].

\section{Quality assessment}

Quality assessment revealed that around $90 \%$ of the included studies showed an overall low risk of bias and only $10 \%$ were at high risk. Specifically, four (17.4\%) of studies were at high risk of bias due to confounding. The higher risk was due to not considering the predefined potential confounders (age at diagnosis, the presence of comorbidities, region, diagnostic year, and tumor stage at diagnosis). In addition, four (17.4\%) of studies were also at high risk of bias due to outcome measurement. In this group, three of the studies at serious risk used self-reported information. Three studies were at high risk of bias due to participant selection and missing data and reported results. The quality appraisal of all the included studies is shown in Fig. 2.

\section{Discussion}

This systematic review aimed to describe the use of health services in long-term ( $\geq 5$ years) breast cancer survivors. A total of 23 observational studies were included that reported data on the health services used by women surviving at least 5 years after their breast cancer diagnosis. Despite the heterogeneity among studies, about half of them reported that breast cancer survivors visit a medical provider at least once a year but that their main medical provider was not a primary healthcare professional, as recommended in the breast cancer survival guidelines [16-18]. In addition, some studies suggested that the number of visits to healthcare services and interventions decreased over time. Results on preventive care were contradictory, with some studies reporting overuse of surveillance tests $[33,35,43]$ and others underuse [31, 39, 46]. However, the studies differed in how they assessed the frequency of the use of preventive care, hampering clear conclusions on the current situation in breast cancer survivors.

No previous systematic reviews were found describing health services use in long-term breast cancer survivors ( $\geq 5$ years). The review by Chopra provides useful information about patterns of health care services use but was not based on long-term survivors [51]. Like our results, those of that review showed substantial variations in terms of the frequency, length, and intensity of follow-up. The authors concluded that breast cancer survivors report a lack of psychosocial support and information on the comorbidities resulting from their cancer treatment.

Regarding the frequency of health services use, 10 studies included in this systematic review [28, 33, 35, 37-39, 41, 42, $47,50]$ showed results in agreement with the breast cancer Survivorship Care Guidelines as they reported that breast cancer survivors visited a healthcare provider at least once a year [16-18]. These guidelines also recommend that from the fifth year after the completion of cancer treatment, the primary care clinician should be the professional in charge of breast cancer survivors' health. We found, however, that only 6 out of 10 studies reported more visits to primary care clinicians than to specialized care [33, 35, 37, 38, 50]. Several factors support the idea of increasing and encouraging primary care utilization. One is that it facilitates coordinated care and reduces specialist visits [52]. Primary care as the axis of prevention and health promotion activities also provides a holistic approach, guaranteeing better coverage of all the recommended preventive activities [16-18]. Health providers in specialized care, on the other hand, are less aware of the preventive recommendations in this population, but they still have to provide these recommendations and receive appropriate training 
Fig. 2 Risk of bias assessment of included studies according to the ROBINS-I assessment tool
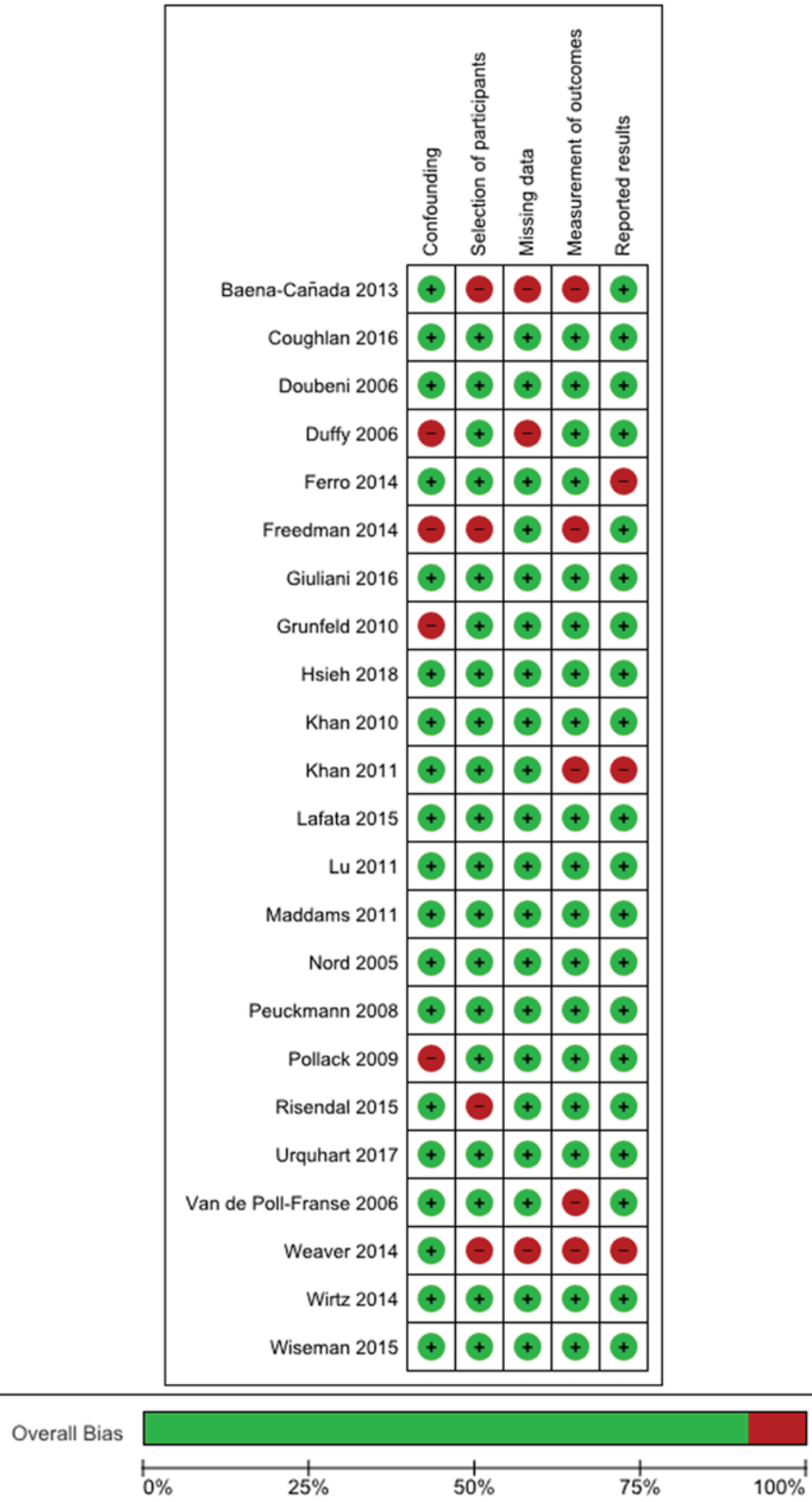

Yes (low risk)

No (high risk) regarding survival care. Although these 10 studies were conducted in different countries, most of them were deemed to be of moderate or high quality. Also, they reported similar results, and therefore, there is sufficient evidence to support the recommendation of 1-year follow-up visits performed by primary care officers.

Watson et al. [53] reported that health promotion advice helps to mitigate the long-term effects of breast cancer, 
improve comorbid conditions, and potentially increase survival. In agreement with these authors, eight studies included in this review reported similar results in breast cancer survivors $[31,36,38,41,45,46,48,49]$. In addition, three of them [35, $44,49]$ stated that breast cancer survivors were more satisfied when the health promotion advice was given by primary health professionals. Overall, the available evidence indicates that primary healthcare providers should place greater emphasis on post-treatment symptoms to make patients aware of them and provide advice on their management. To do this, the best option seems to be the shared care model since it can facilitate a continued relationship in terms of updates and changes in surveillance recommendations and potential late effects [53, 54]. Primary care clinicians should consult with the cancer treatment team to ensure evidence-based and well-coordinated care.

Surveillance mammography has been recommended for both the early detection of local recurrences as well as the screening of de novo primary breast cancer [16-18]. The results of our review suggest that improvements should be made to increase adherence to this recommendation given that underuse of mammography has been described in four highquality studies [30, 35, 39, 45]. Furthermore, our results suggest that clinicians should refer women for an annual mammogram rather than to other screening strategies, unless the patient is at high risk of developing de novo cancer or recurrence [16-18]. Heterogeneity in adherence to mammography as a recommended surveillance test can be due to national screening policies, professional decisions, or structural barriers. Thus, efforts need to be made to ensure an annual mammogram in long-term survivors and avoid losses to follow-up $[55,56]$.

In terms of other preventive care, the results of two highquality studies included $[31,43]$ show that laboratory tests and imaging scans are more frequent in breast cancer survivors than in the general population, probably related to comorbidities and surveillance of cancer recurrence surveillance. According to expert opinion, routine laboratory or imaging tests to evaluate breast cancer recurrence are not recommended in asymptomatic patients, while screening for other cancers and preventive diseases should be done as in patients in the general population [16-18]. These results support active surveillance through primary care medical providers in coordination with specialized care for the correct management of comorbidities and to raise awareness of the appropriate prescription of laboratory tests and imaging scans.

This review has some limitations. Some studies used selfreported information on health services use and in the identification of breast cancer survivors [28, 29, 32, 33, 36, 41, 44, 48-50]. This could lead to a reporting bias, specifically a memory bias, which tends to overestimate or underestimate outcomes in survivors. Other limitations are the differences between the populations, survival periods, age at diagnosis, and year of diagnosis included in each study. All studies included survivors of 5 years or more, but the periods were not restricted at any point, so the survival periods ranged from 5 to 16 years. We could not analyze the differences between longer and shorter survivorship, which could also help to develop specific protocols. Survival can lead to a selection bias, since longer survival is related to better quality of life [39, 41, 44, $49,50]$ and consequently to fewer visits to health services. Advances in breast cancer treatment need to be taken into account, as some women included in this review did not receive the most recent therapies and might have experienced an increased number of adverse effects or shown a different pattern of health services use related to updating of survival guidelines. Finally, we found no RCTs that tested interventions in breast cancer survivors regarding the management of long-term late effects and comorbidities due to cancer treatment.

This is the first systematic review to include long-term breast cancer survivors (at least 5 years of survivorship), revealing heterogeneity in follow-up activities and areas for improvement. Further research with mixed methodology is needed to identify the sources of the variations, professionals' perceptions, and patients' needs. Such knowledge would generate opportunities to mitigate current barriers to health services and to structure shared follow-up models.

\section{Conclusions}

The literature on the use of health care services among longterm breast cancer survivors shows substantial heterogeneity in methods to evaluate the follow-up characteristics in this specific population. The results of this review help to support the need for actions to improve active surveillance through primary care providers in coordination with specialized care through shared care models of follow-up. Furthermore, this approach can be strongly recommended due to the high quality of the studies in favor of the latter. Our results could also help to standardize the appropriate management of breast cancer survivors and decision-makers in different contexts to adapt their guidelines and clinical protocols. More research is still needed to provide specific data, but this review helps to support primary care providers as the main health providers for this group of women with particular needs.

Acknowledgments Anna Jansana is a Ph.D. candidate at the Methodology of Biomedical Research and Public Health program, Universitat Autònoma de Barcelona (UAB), Barcelona, Spain.

The authors acknowledge the dedication and support of the SURBCAN Study Group (alphabetical order):

Hospital del Mar Medical Research Institute (IMIM), Barcelona: Mercè Abizanda, Mercè Comas, Laia Domingo, Talita Duarte, Anna Jansana, Javier Louro, Maria Sala. 
Hospital Costa del Sol, University of Málaga: María del Carmen Martínez, Cristóbal Molina, María del Carmen Padilla, Maximino Redondo.

EpiChron Group of Chronic Disease Research, Zaragoza: Antonio Gimeno, Manuela Lanzuela, Beatriz Poblador, Alexandra Prados.

Primary Care Research Unit. Gerencia de Atención Primaria, Madrid: Ángel Alberquilla, Isabel del Cura, Antonio Díaz, Teresa Sanz.

Miguel Servet Health services and Cronicity Research Group, Navarra: Javier Baquedano, Rosana Burgui, Berta Ibáñez, María Concepción Moreno.

Funding This study was funded by the Carlos III Health Institute through the project "PI16/0024" and "PI15/00098"and co-funded by FEDER (European Regional Development Fund/European Social Fund), and by the Research Network on Health Services in Chronic DiseasesREDISSEC (RD16/0001/0013).

\section{Compliance with ethical standards}

Conflict of interest The authors declare that they have no conflict of interest. This protocol is registered in PROSPERO (2018:CRD42018089309).

Ethical approval This article does not contain any studies with human participants or animals performed by any of the authors.

Informed consent For this type of study, formal consent is not required.

Open Access This article is distributed under the terms of the Creative Commons Attribution 4.0 International License (http:// creativecommons.org/licenses/by/4.0/), which permits unrestricted use, distribution, and reproduction in any medium, provided you give appropriate credit to the original author(s) and the source, provide a link to the Creative Commons license, and indicate if changes were made.

\section{References}

1. Cardoso F, Harbeck N, Barrios C, Bergh J, Cortés J, El Saghir N, et al. Research needs in breast cancer. Ann Oncol. 2017;28(2):20817.

2. Engholm G, Ferlay J, Christensen N, Bray F, Gjerstorff ML, Klint A, et al. NORDCAN-a Nordic tool for cancer information, planning, quality control and research. Acta Oncol. 2010;49(5):725-36.

3. Noone A, Howlader N, Krapcho M, Miller D, Brest A, Yu M, Ruhl J, SEER Cancer Statistics Review, et al., 1975-2015. National Cancer Institute. Bethesda. Available at: https://seer.cancer.gov/ csr/1975_2015/. Accessed 21 Feb 2019.

4. Cancer Research UK. Breast cancer survival statistics. Available at: https://www.cancerresearchuk.org/. Accessed 20 Feb 2018.

5. Wang W, Tan S, Chow W. The impact of mammographic breast cancer screening in Singapore: a comparison between screendetected and symptomatic women. Asian Pac J Cancer Prev. 2011;12(10):2735-40.

6. Hugh-Yeun K, Kumar D, Moghaddamjou A, Ruan J, Cheung W. Young adult cancer survivors' follow-up care expectations of oncologist and primary care physicians. J Cancer Surviv. 2017;11: 295-301.

7. Boyle P, Levin B. World Cancer Report 2008. International Agency for Research on Cancer (IARC). Geneva: 2010.
8. Erikson C, Salsberg E, Forte G, Bruinooge S, Goldstein M. Future simply and demand for oncologist: challenges to assuring access to oncology services. J Oncol Pract. 2007;3(2):79-86.

9. Miller K, Siegel R, Lin C, Mariotto A, Kramer J, Rowland J, et al. Cancer treatment and survivorship statistics, 2016. CA Cancer J Clin. 2016;66(4):271-89.

10. Surveillance, Epidemiology, and End Results (SEER) Program. SEERStat Database: Incidence-SEER-9 regulations. National Cancer Institute, Division of Cancer Control and Population Sciences. Bethesda: 2003. Available at: https://seer.cancer.gov/ Accessed July 20, 2018.

11. Schmitz K, Speck R, Rye S, DiSipio T, Hayes S. Prevalence of breast cancer treatment sequelae over 6 years of follow-up: the pulling through study. Cancer. 2012;118(8 Suppl):2217-25.

12. Rubinstein E, Miller W, Hudson S, Howard J, O'Malley D, Tsui J. Lee, $\mathrm{H}$ et al. Cancer survivorship care in advanced primary care practices. A qualitative study of challenges and opportunities. JAMA Intern Med. 2017;177(12):1726-32.

13. Deckx L, Van den Akker M, Metsemakers J, Knottnerus A, Schellevis F, Buntinx F. Chronic diseases among older cancer survivors. J Cancer Epidemiol. 2012;2012:206414.

14. Boekel NB, Schaapveld M, Gietema JA, et al. Cardiovascular morbidity and mortality after treatment for ductal carcinoma in situ of the breast. J Natl Cancer Inst. 2014;106(8). https://doi.org/10.1093/ jnci/dju156.

15. National Cancer Institute. Public Health Research and Cancer. Bethesda. Available at: https://www.cancer.gov/research/areas/ public-health\#opportunities. Accessed 25 Jan 2019.

16. Runowicz C, Leach C, Henry N, Henry K, MEckey H, CowensAlvarado R, et al. American Cancer Society/American Society of Clinical Oncology breast Cancer survivorship Cre guideline. CA Cancer J Clin. 2016;66(1):43-73.

17. Alba E, Cruz J, Barón F, Rodríguez X, Blasco A, Escobar Y, et al. Monográfico SEOM de largos supervivientes en cáncer. Madrid: Sociedad Española de Oncología Médica; 2012. Available at: https:// seom.org/seomcms/images/stories/recursos/infopublico/publicaciones/ MONOGRAFICO_SEOM_LARGOS_SUPERVIVIENTES_I.pdf. Accessed 20 April 2018.

18. Hewitt M, Greenfield S, Stovall E. Delivering cancer survivorship care - from cancer patient to cancer survivor: lost in transition. Washington DC: The National Academies Press; 2006. p. 187-321.

19. Snyder C, Frick K, Herbert R, Clackford A, Neville BA, Lemke $\mathrm{KW}$, et al. Comorbid condition care quality in cancer survivors: role of primary care and specialty providers and care coordination. J Cancer Surviv Res Pract. 2015;9:641-9.

20. Snyder C, Frick K, Herbert R, Clackford A, Neville B, Wolff A, et al. Quality of care for comorbid conditions during the transition to survivorship: differences between cancer survivors and noncancer controls. J Clin Oncol L. 2013;31(9):1140-8.

21. Blanchard C, Denniston M, Baker F, Ainsworth S, Courneya K, Hann D, et al. Do adults change their lifestyle behaviours after a cancer diagnosis? Am K Health Behav. 2003;27:246-56.

22. Kerrigan D, Waters P, Ryan M, Irfan M, Hanaghan J, Khan W, et al. Follow-up arrangements for breast cancer patients; is it appropriate to transfer surveillance to general practitioners? Ir Med J. 2014;107(9):273-5.

23. Hudson S, Ohman-Strickland P, Bator A, O'Malley D, Gundersen $\mathrm{D}$, Lee HS, et al. Breast and prostate cancer survivors' experiences of patient-centered cancer follow-up care from primary care physicians and oncologists. J Cancer Surviv. 2016;10(5):906-14.

24. Higgins J, Altman D, Gøtzsche P, Jüni P, Moher D, Oxman A, et al. The Cochrane Collaboration's tool for assessing risk of bias in randomised trials. BMJ. 2011;343:d5928.

25. Moher D, Shamseer M, Clarke M, Ghersi D, Liberati A, Petticrew $\mathrm{M}$, et al. Preferred reporting items for systematic review and meta- 
analysis protocols (PRISMA-P) 2015 statement. Syst Rev BMJ. 2015;349:g7647.

26. Romond E, Perez E, Bryant J, Suman V, Geyer C, Davidson N, et al. Trastuzumab plus adjuvant chemotherapy for operable HER-2 positive breast cancer. N Engl J Med. 2005;353(16):1673-84.

27. Sterne J, Hernán M, Reeves B, Savović J, Berkman N, Viswanathan $\mathrm{M}$, et al. ROBINS-I: a tool for assessing risk of bias in nonrandomised studies of interventions. BMJ. 2016;355. https://doi. org/10.1136/bmj.i4919.

28. Baena-Cañada J, Ramírez-Daffós P, Cortés-Carmona C, RosadoVarela P, Nieto-Vera J, Benítez-rodríguez E. Follow-up long longterm survivors of breast cancer in primary care versus specialist attention. Fam Pract. 2013;30:525-32.

29. Coughlan D, Doherty E, Frick K, Ward P, O'Neill C. Healthcare utilisation among cancer survivors over 50 years of age. Ir Med J. 2016;109(2):359.

30. Doubeni C, Field S, Ulcickas Yood M, Rolnick S, Quessenberry C, Fouayzi $\mathrm{H}$, et al. Patterns and predictors of mammography utilization among breast cancer survivors. Cancer. 2006;106(11):2482-8.

31. Duffy C, Clark M, Allsworth J. Health maintenance and screening in breast cancer survivors in the United States. Cancer Detect Prev. 2006;30(1):52-7.

32. Ferro $\mathrm{T}$, Aliste L, Valverde $\mathrm{M}$, Fernández $\mathrm{M}$, Ballano $\mathrm{C}$, Borràs $\mathrm{J}$. Health status and health resource use among long-term survivors of breast colorectal and prostate cancer. Gac Sanit. 2014;28(2):12936.

33. Freedman A, Keating M, Pace L, Lii J, McCarthy E, Schonberg M. Use of Surveillance Mammography Among Older Breast Cancer Survivors by Life Expectancy. J Clin Oncol. 2017;35(27):3123-30.

34. Giuliani O, Mancini S, Puliti D, Caranci N, Ravaioli A, Vattiato R, et al. Patterns and determinants of receipt of follow-up mammography and/or clinical examination in a cohort of Italian breast cancer survivors. Breast Cancer Res Treat. 2016;158(3):543-51.

35. Grunfeld E, Hodgson DC, Del Giudice ME, Moineddin R. Population-based longitudinal study of follow-up Care for Breast Cancer Survivors. J Oncol Pract. 2010;6(4):174-81.

36. Khan N, Carpenter L, Watson E, Rose P. Cancer screening and preventative care among long-term cancer survivors in the United Kingdom. Br J Cancer. 2010;102(7):1085-90.

37. Khan N, Watson E, Rose P. Primary care consultation behaviours of long-term, adult survivors of cancer in the UK. Br J Gen Pract. 2011;61(584):197-9.

38. Lafata J, Salloum R, Fishman P, Ritzwoller D, O'Keeffe-Rosetti M, Hornbrook M. Preventive care receipt and office visit use among breast and colorectal Cancer survivors relative to age- and gendermatched Cancer-free controls. J Cancer Surviv. 2015;9(2):201-7.

39. Lu W, Jansen L, Schaapveld M, Baas PC, Wiggers T, De Bock GH. Underuse of long-term routine hospital follow-up care in patients with a history of breast cancer? BMC Cancer. 2011;11:279.

40. Maddams J, Utley M, Møller H. A person-time analysis of hospital activity among cancer survivors in England. Br J Cancer. 2011;105(1):S38-45.

41. Nord C, Mykletun A, Thorsen L, Bjøro T, Fosså SD. Self-reported health and use of health care services in long-term cancer survivors. Int J Cancer. 2005;114(2):307-16.

42. Pollack L, Adamache W, Ryerson A, Eheman C, Richardson L. Care of long-term cancer survivors: physicians seen by Medicare enrollees surviving longer than 5 years. Cancer. 2009;115(22): 5284-95.
43. Risendal B, Sedjo R, Giuliano A, Vadaparampil S, Jacobsen P, Kilbourn K, et al. Surveillance and beliefs about follow-up care among long-term breast cancer survivors: a comparison of primary care and oncology providers. J Cancer Surviv. 2016;10(1):96-102.

44. Urquhart R, Lethbridge L, Porter G. Patterns of cancer Centre follow-up care for survivors of breast, colorectal, gynecologic, and prostate cancer. Curr Oncol. 2017;24(6):360-6.

45. Wirtz H, Boudreau D, Gralow J, Barlow W, Gray S, Erin J, et al. Factors associated with long-term adherence to annual Surveillance mammography among breast Cancer survivors. Breast Cancer Res Treat. 2014;143(3):541-50.

46. Hsieh E, Wang Q, Zhang R, Niu X, Xia W, Fraenkel L, et al. Vertebral fractures among breast cancer survivors in China: a cross-sectional study of prevalence and health services gaps. BMC Cancer. 2018;18(1):104.

47. Peuckmann V, Ekholm O, Sjøgren P, Rasmussen N, Christiansen P, Møller S, et al. Health care utilisation and characteristics of longterm breast cancer survivors: nationwide survey in Denmark. Eur J Cancer. 2009;45(4):625-33.

48. Poll-Franse L, Mols F, Vingerhoets A, Voogd A, Roumen R, Coebergh J. Increased health care utilisation among 10-year breast cancer survivors. Support Care Cancer. 2005;14:436-43.

49. Weaver K, Aziz N, Arora N, Forsythe L, Hamilton A, OakleyGirvan I, et al. Follow-up care experiences and perceived quality of care among long-term survivors of breast, prostate, colorectal, and gynecologic cancers. J Oncol Pract. 2014;10(4):e231-9.

50. Wiseman K, Bishop D, Shen Q, Jones R. Survivorship care plans and time since diagnosis: factors that contribute to who breast cancer survivors see for the majority of their care. Support Care Cancer. 2015;23(9):2669-76.

51. Chopra I, Chopra A. Follow-up care for breast cancer survivors: improving patient outcomes. Patient Relat Outcome Meas. 2014;5: 71-85.

52. LaGrandeur W, Armin J, Howe CL, Ali-Akbarian L. Survivorship care plan outcomes for primary care physicians, cancer survivors, and systems: a scoping review. J Cancer Surviv. 2018;12(3):33447.

53. Watson E, Rose P, Loftus R, Devane C. Cancer survivorship: the impact on primary care. Br J Gen Pract. 2011;61(592):e763-5.

54. Bucchi L, Belli P, Benelli E, Bernardi D, Brancato B, Calabrese M, et al. Recommendations for breast imaging follow-up of women with a previous history of breast cancer: position paper from the Italian Group for Mammography Screening (GISMa) and the Italian College of Breast Radiologists (ICBR) by SIRM. Radiol Med. 2016;121(12):891-6.

55. Senkus E, Kyriakides S, Ohno S, Penault-Llorca F, Poortmans P, Rutgers E, et al. Primary breast cancer: ESMO clinical practice guidelines for diagnosis, treatment and follow-up. Ann Oncol. 2015;26:8-30.

56. Barnadas A, Algara M, Córdoba O, Casas A, Gonzalez M, López T, et al. Recomendaciones para el seguimiento de las mujeres supervivientes de cáncer de mama. Sociedad Española de Oncología Médica. 2017. Available at: http://sespm.es/archivos/ Recomendaciones-para-Seguimiento-de-las-MujeresSupervivientes-Cancer-de-Mama.pdf. Accessed 18 May 2018.

Publisher's note Springer Nature remains neutral with regard to jurisdictional claims in published maps and institutional affiliations. 